\title{
Numbers on maps as a new cartographic technique: helpful or harmful for users? An empirical study
}

\author{
Korycka-Skorupa, Jolanta ${ }^{\text {a, * }}$ \\ ${ }^{a}$ POLAND, University of Warsaw, Faculty of Geography and Regional Studies, Department of Geoinformatics, Cartography and \\ Remote Sensing, j.skorupa@uw.edu.pl \\ * Corresponding author
}

Keywords: Cartographic visualization, Cartographic representation techniques, Quantitative data, Numbers, Graduated numbers, Proportional symbols

\begin{abstract}
:
The huge popularity of the map as a medium of information transfer means that the number of map makers and map users is constantly increasing. In an era of GIS technology, open-source software, and wide access to spatial data, map making may seem simple, and the skills required available to almost everyone; however, the simplicity is only apparent. In fact, the creation of an efficient and effective map is extremely difficult, labour-intensive, and requires a good knowledge of the principles of cartographic representations.

Map creation requires the use of various graphic solutions, and various cartographic representation techniques can be used. The most commonly used 'graphic alphabet' is by Jacques Bertin, i.e., visual variables. For the presentation of quantitative data expressed by numerical values, it is most appropriate to use visual variables of a size or value that correctly reflect the differences in the statistical data. These variables most often form the basis for the cartographic presentation of quantitative data.
\end{abstract}

An effective, efficient, and proper map, according to J. Bertin (1983), is one that generalizes information and displays it using appropriately selected graphic symbols. Sometimes more variables are used to improve and highlight the information presented on the map, i.e., when size and value are used simultaneously - it is called redundancy (Slocum et all 2010, Morrison, Watson, Morrison 2015).

Currently, there is a tendency to show a lot of information on maps, using the maximum amount of precision and detail. This often produces the opposite result: the map becomes illegible, does not explain phenomena, and misleads the user. These facts prompted us to look at a new cartographic solution which increasingly appears on maps: numbers used for presenting quantitative information (Brewer 1998).

This paper presents empirical research on the numbers (Fig. 1a), graduated numbers (Fig. 1b), and proportional symbols (Fig. 1c) used in cartographic representation techniques for quantitative data. The research was conducted in Poland in 2019. There were over 600 participants, high school students from general and technical schools. They were asked to analyse maps and solve different types of tasks. The time and correctness of response were examined to look for differences between the cartographic representations.

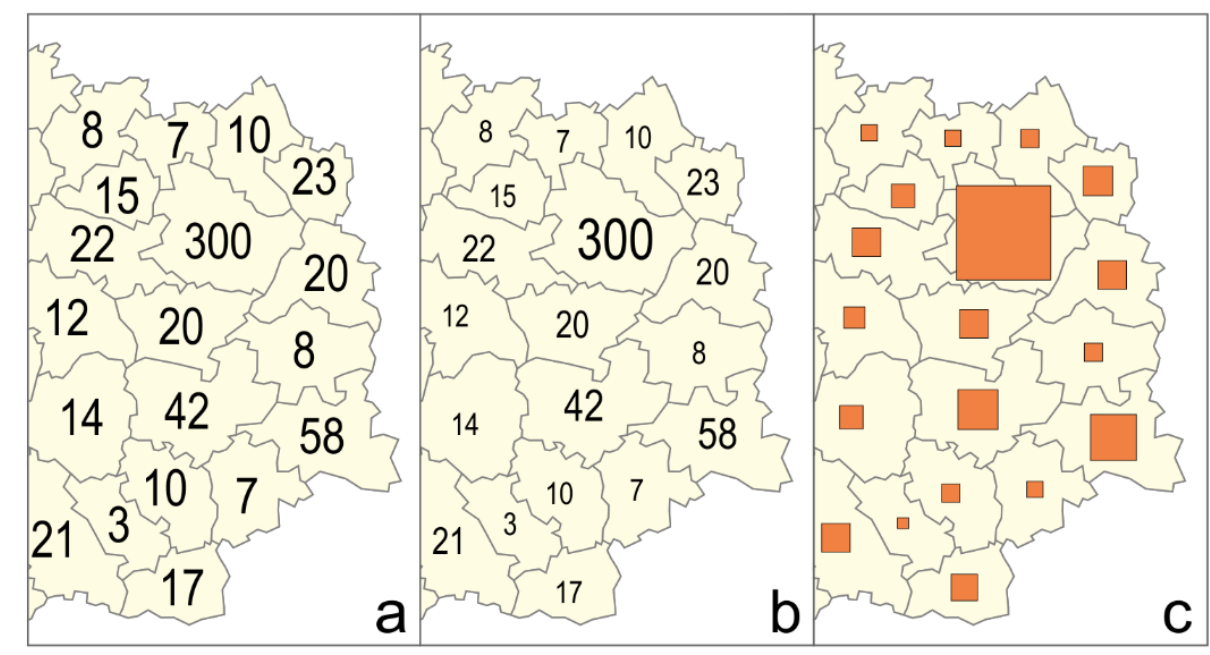


Figure 1. (a) numbers, (b) graduated numbers, and (c) proportional symbols used in cartographic representation techniques of quantitative data.

The research asked whether numbers or graduated numbers used on maps could be treated as an independent cartographic representation technique, or if they should be used only as a complement to other representation techniques. It was very interesting to learn which cartographic representation technique is more useful and effective: numbers, graduated numbers, or proportional symbols (squares).

The research verified whether numbers and graduated numbers work better when reading a map at a detailed level (e.g. comparing values in two or three neighbouring units), than when reading the map at a general level. Numbers and graduated numbers seem to be very close to proportional symbols. It was also interesting to learn if these were legible enough to interpret the map properly, and therefore if they are helpful or harmful for map users.

This work was supported by the Polish National Science Centre [Grant number UMO-2016/23/B/HS6/03846], "Evaluation of cartographic presentation methods in the context of map perception and effectiveness of visual transmission." 